\title{
The Important Questions of Statistical Theory of the Crystals' Thermal and Kinetic Properties
}

\author{
${ }^{1}$ Lviv Polytechnic National University, Lviv, Ukraine, e-mail: jabudjak@ukr.net \\ ${ }^{2}$ Krakow Polytechnic, Krakow, Poland, e-mail: tadeuszwaclawski00@gmail.com
}

\begin{abstract}
In this paper, the short algorithmic formulas for computations of the crystals thermal and kinetic properties are given. These formulas are fulfilled the case of isotropic crystals for any one of dispersion laws of current carriers, where these carriers can be scattered by all kinds of the crystal lattice defects. In the paper, these algorithmic formulas were used to calculations the important properties of crystals with the nonparabolic Kane's dispersion law of current carriers. Here, the passing from these non-parabolic dispersion law crystals to the parabolic dispersion law crystals was also described.
\end{abstract}

Keywords: Gibbs potential, chemical potential, electrical conductivity, thermal conductivity, tensor.

Received 03.12.2019; accepted for publication 15.03.2020.

\section{Thermal properties of the charge carriers gas in crystals}

At the equilibrium state, the free charge carriers are moving chaotically, their average energy remains constant and the entropy of all the set of current carriers has its maximum value. This is the thermodynamic equilibrium gas of the charge carriers. This gas is described by the Gibbs grand thermodynamic potential $\Omega$. This potential, as it was shown in the cited literature [1-5], is described by the following general algorithmic formula:

$$
\Omega=-V \int_{0}^{\infty} \frac{G(\varepsilon)}{\left.\exp \left(\frac{\varepsilon-\mu}{k T}\right)+1\right)} d \varepsilon=-V \int_{0}^{\infty} G(\varepsilon) f_{0}(\varepsilon) d \varepsilon \text { (1тh) }
$$

In this formula, $V$ is the crystal volume, $f_{0}(\varepsilon)$ is the known Fermi-Dirac function, $G(\varepsilon)=\int_{0}^{\varepsilon} g(\varepsilon) d \varepsilon$, and $g(\varepsilon)=\frac{d G(\varepsilon)}{d \varepsilon}$ is the density of the energy states (DOS) of charge carriers lying in allowed band, $\varepsilon$ is the energy of quantum particle, it depends on the quasi-momentum vector $\vec{p}$. The function $\varepsilon(\vec{p})$ is called the dispersion law. According to the quantum theory laws, the exact dispersion relation is a periodic and even function of the vector $\vec{p}$.

The thermal properties of quantum gases, via the Gibbs grand thermodynamic potential $\Omega$ (1th), are described by the following set of general algorithmic formulas:

$$
\begin{gathered}
U_{n}=\Omega_{n}-\left(\frac{d \Omega_{n}}{d \mu}\right)_{T} \mu-\left(\frac{d \Omega_{n}}{d T}\right)_{\mu} T=V \cdot n\left(\mu^{\bullet}, T\right) \cdot\left(\frac{\Omega}{V \cdot J\left(0, \mu^{\bullet}, T\right)}+\frac{J\left(1, \mu^{\bullet}, T\right)}{J\left(0, \mu^{\bullet}, T\right)}\right), \\
H_{n}=-\left(\frac{d \Omega_{n}}{d \mu}\right)_{T} \mu-\left(\frac{d \Omega_{n}}{d T}\right)_{\mu} T=V \cdot n\left(\mu^{\bullet}, T\right) \cdot\left(\frac{J\left(1, \mu^{\bullet}, T\right)}{J\left(0, \mu^{\bullet}, T\right)}\right),
\end{gathered}
$$




$$
\begin{gathered}
F_{n}=\Omega_{n}-\left(\frac{d \Omega_{n}}{d \mu}\right)_{T} \mu=V \cdot n\left(\mu^{\bullet}, T\right) \cdot\left(\frac{\Omega}{V \cdot J\left(0, \mu^{\bullet}, T\right)}+\mu\right), \\
G_{n}=-\left(\frac{d \Omega_{n}}{d \mu}\right)_{T} \mu=V \cdot J\left(0, \mu^{\bullet}, T\right) \cdot \mu=V \cdot n\left(\mu^{\bullet}, T\right) \cdot \mu, \\
\Omega=-V \int_{0}^{\infty} \frac{G(\varepsilon)}{\left(\exp \left(\frac{\varepsilon-\mu}{k T}\right)+1\right)} d \varepsilon=-V \int_{0}^{\infty} G(\varepsilon) f_{0}(\varepsilon) d \varepsilon, \\
S_{n}=-\left(\frac{d \Omega_{n}}{d T}\right)_{\mu}=\frac{U_{n}-F_{n}}{T}=\frac{V}{T} \cdot n\left(\mu^{\bullet}, T\right) \cdot\left(\frac{J\left(1, \mu^{\bullet}, T\right)}{J\left(0, \mu^{\bullet}, T\right)}-\mu\right), \\
P V=-\Omega_{n}=k T \ln \left(\Xi_{n}\right)=N_{n} \frac{J(G(\varepsilon))}{J(g(\varepsilon))^{\prime}} \\
\frac{N_{n}}{V}=-\frac{1}{V}\left(\frac{d \Omega_{n}}{d \mu}\right)_{T}=\int_{0}^{\infty} G(\varepsilon)\left(-\frac{d f_{0}(\varepsilon)}{d \varepsilon}\right) d \varepsilon=J\left(0, \mu^{\bullet}, T\right)=n\left(\mu^{\bullet}, T\right) .
\end{gathered}
$$

In these formulas, $n\left(\mu^{\bullet}, T\right)$ denotes the concentration of crystal current carriers' gas, and besides, the following general notations were used for an integral:

$$
J\left(i, \mu^{\bullet}, T\right)=\int_{0}^{\infty} \varepsilon^{i} \cdot G(\varepsilon)\left(-\frac{d f_{0}(\varepsilon)}{d \varepsilon}\right) d \varepsilon,
$$

where $f_{0}(\varepsilon)=\left(\exp \left(\frac{\varepsilon-\mu}{k T}\right)+1\right)^{-1}$ is the known FermiDirac function.

Definitions and physical meaning of the thermodynamic potentials (2th)-(10th) are given in the cited works [1-5].

\section{Kinetic properties of the conducting crystals}

Kinetic properties of the conducting crystals are determined by the concentration of the free charge carriers in the crystals and by the character of their motion throughout the crystal interstitial site.

In equilibrium state, the free charge carriers are moving chaotically, their average energy is conserved, and the entropy of the system of these carriers obtains its maximum value. This is an equilibrium gas of the charge carriers. Necessary conditions for the system to be in this state are the constant values of temperature and chemical potential in all its points. If even one of these conditions is not satisfied, then there the non-equilibrium processes result in the crystal. These non-equilibrium processes are trying to bring the system to the thermodynamic equilibrium state.

To remove the crystal out from equilibrium, the electric field $\vec{E}$, the temperature gradient $\nabla_{\vec{r}} T$ or the chemical potential gradient $\nabla_{\vec{r}} \mu$ must exists in the crystal. These fields may exist simultaneously in the crystal. In the presence of these fields in the crystal there are the fluxes of mass, energy and charge - these are described by the first and second laws of nonequilibrium thermodynamics:

$$
\frac{d U_{e}}{d t}=-\operatorname{div} \vec{q}+\vec{\jmath} \vec{E}
$$

\section{I (the first law of nonequilibrium} thermodynamics)

$$
\frac{d S_{e}}{d t}=\frac{1}{T}\left(\vec{\jmath} \vec{E}-\frac{\vec{q} \nabla_{\vec{r}} T}{T}\right) .
$$

\section{II (the second law of nonequilibrium thermodynamics)}

In the above laws, $\vec{\jmath}, \vec{q}$ are the vectors of the electric current density and heat flow, and $U_{e}, S_{e}$ are respectively the internal energy and entropy of the system.

In statistical physics it was shown that when there is an increase of the entropy in the thermodynamic system, the processes of heat and electric charge transport (processes of heat and electric conduction) occur. Conversely, when the processes of heat and electric charge transport occur in the system, its entropy inreases. Thus, we have:

$$
\frac{d S_{e}}{d t}=\frac{1}{T}\left(\vec{\jmath} \vec{E}-\frac{\vec{q} \nabla_{\vec{r}} T}{T}\right) \geq 0 .
$$

In nonequilibrium thermodynamics, it was shown that under the above conditions there the electric charge and heat transport processes begin to exist in the crystal. These processes are described by the following generalized electrical and heat conduction equations:

$$
\begin{aligned}
& \vec{\jmath}=\left(\sigma_{i k}(\vec{B})\right) \vec{E}-\left(\beta_{i k}(\vec{B})\right) \nabla_{\vec{r}} T, \\
& \vec{q}=\left(\gamma_{i k}(\vec{B})\right) \vec{E}-\left(h_{i k}(\vec{B})\right) \nabla_{\vec{r}} T .
\end{aligned}
$$

Equations (1), (2) - these are well known in nonequilibrium thermodynamics generalized equations of electrical and heat conduction. They describe the response of conducting medium to the action of the 
electric field, temperature gradient and magnetic field. Coefficients in these equations, $\left(\sigma_{i k}(\vec{B})\right),\left(\beta_{i k}(\vec{B})\right),\left(\gamma_{i k}(\vec{B})\right),\left(h_{i k}(\vec{B})\right)$ - these are the tensors of kinetic coefficients. These tensors describe numerous matter properties of the conducting medium (i. e., this crystal).

The tensors of kinetic coefficients and the coefficients of different phenomena given in this work are calculated with the use Gibbs grand canonical thermodynamic potential $\Omega$. This potential is detailed described in the works [1-5]. It is given as:

$$
\Omega=-2 V \int_{0}^{\infty} \frac{G(\varepsilon)}{\left(\exp \left(\frac{\varepsilon-\mu-\Delta \mu_{p}}{k T}\right)+1\right)} d \varepsilon
$$

In this formula, $\varepsilon$ is the charge carriers' energy dispersion law, and $\Delta \mu(\vec{p})$ is the change in the one particle chemical potential by the action of these perturbations. When the perturbations are absent, $\Delta \mu(\vec{p})=0$ and then, this thermodynamic potential will be equal to the classical Gibbs grand canonical thermodynamic potential.

The value $\Delta \mu(\vec{p})$ was calculated in the works [1-5], where it was shown that $\Delta \mu(\vec{p})$ is an odd function of the vector $\vec{p}$ and it has an analytical dependence on the dispersion law, chemical potential and magnetic induction vector $\vec{B}$.

The statistical equations (1), (2), that were obtained with the use potential (3), via linear transformation may take the following form:

$$
\begin{aligned}
& \vec{E}=\left(\rho_{i k}(\vec{B})+R_{i k} \delta_{i k l} B_{l}\right) \vec{\jmath}+\left(\alpha_{i k}(\vec{B})+N_{i k} \delta_{i k l} B_{l}\right) \nabla_{\vec{r}} T, \\
& \vec{q}=\left(\pi_{i k}(\vec{B})+P_{i k} \delta_{i k l} B_{l}\right) \vec{\jmath}-\left(\chi_{i k}(\vec{B})+S_{i k} \delta_{i k l} B_{l}\right) \nabla_{\vec{r}} T .
\end{aligned}
$$

In these equations, $\delta_{i k l}$ is the Levi-Civita symbol. It has the following properties: $\delta_{i k l}=1$, if " $i k l$ " is an even permutation of numbers "ikl"; $\delta_{i k l}=-1$, if " $i k l$ " is an odd permutation of these numbers; " $i k l "=0$, if any two indices are equal. Besides, in these formulas a summation is over the indices " $l$ ", which appear twice in a single term.

The physical meaning of the statistical tensors in equations (4) and (5) may be cleared up with the use of the number phenomenological laws of non-equilibrium thermodynamics. They describe all set of the galvanomagnetic and thermomagnetic effects in semiconductor crystals. Such analysis of these equations shows that $\left(\rho_{i k}\right),\left(\alpha_{i k}\right),\left(\pi_{i k}\right),\left(\chi_{i}\right)$ - these are respectively, the material tensors of the resistivity, Seebeck effect, Peltier effect and thermal conductivity, and $R_{i k}, N_{i k}, P_{i k}, S_{i k}$ - these are respectively, the coefficients of the Hall effect, Nernst-Ettigshausen effect, Nernst effect, Righi-Leduc effect.

All tensors and coefficients, according to the Onsager principle for the kinetic coefficients, can be only even functions of the induction magnetic vector, and the tensor coefficients $R_{i k}, N_{i k}, P_{i k}, S_{i k}-$ in isotropic crystals - turn into scalars $R, N, P, S$.

Equations (4), (5) show that in the presence of a magnetic field in a crystal, the relativity simple processes of the electric and heat conduction will become more complicated. In this case, the additional transverse galvanomagnetic and thermomagnetic effects there occur. The first effects are determinated by the action of a magnetic field on the omic part of the electrical current, and the second effects - by the action of this field on the thermal part. According to the generalized equation of electrical conduction (1), the electrical current is composed of the omic part (that it is proportional to the electric field strength) and the thermal part (that it is proportional to the temperature gradient).

Therefore, this analysis shows that the generalized electrical and heat conduction equations (4) and (5) of the isotropic crystals may be written in the following form:

$$
\begin{gathered}
\vec{E}=\left(\rho_{i k}(\vec{B})\right) \vec{\jmath}+R(\vec{B})[\vec{B} \times \vec{\jmath}]+\left(\alpha_{i k}(\vec{B})\right) \nabla_{\vec{r}} T+N(\vec{B})\left[\vec{B} \times \nabla_{\vec{r}} T\right], \\
\vec{q}=\left(\pi_{i k}(\vec{B})\right) \vec{\jmath}+P(\vec{B})[\vec{B} \times \vec{\jmath}]-\left(\chi_{i k}(\vec{B})\right) \nabla_{\vec{r}} T+S(\vec{B})\left[\vec{B} \times \nabla_{\vec{r}} T\right] .
\end{gathered}
$$

In these equations, the vector products are denoted by the square brackets.

All these kinetic tensors and coefficients in these equations have pragmatic significance for modern solidstate electronics, because they describe a number of the crystal properties used in the fabrication of solid state instruments and systems.
The formulas to calculations the tensors and kinetic coefficients of equations (6), (7) for an arbitrary strength of the magnetic induction vector in a crystal have very complicated structure and symmetry.

When the magnetic induction vector is agreeing in its direction with the one major axis of a crystal energy valley, then these formulas will have the simplest 
structure and symmetry. In this case, all symmetric tensors become diagonal and antisymmetric tensors obtain their simplest form. In the isotropic crystals every axis may be taken as a major axis.

For example, suppose that indices "123" denote the coordinate axes of Cartesian system and the magnetic induction vector is agreeing in direction with the "3"axis. This axis is aligned with the major axis of a crystal energy valley and it is normal to the vectors $\overrightarrow{E_{d}}$ and $\nabla_{\vec{r}} T$ (where $\quad \overrightarrow{E_{d}}=\vec{E}-\left(\frac{k}{z e}\right)\left(\frac{\varepsilon-\mu}{k T}\right) \nabla_{\vec{r}} T$ ). Under these conditions, all tensors and coefficients in equations (6), (7) depend on the induction vector $B_{3}$, then the symmetric tensors become diagonal as well as antisymmetric tensors will have their simplest form. In this case, taking into consideration the structure of the tensors in these equations, we find the following formulas for these tensors and coefficients:

$$
\begin{gathered}
\left(\rho_{i j}\left(B_{3}\right)\right)=\left(\rho_{i i}\left(B_{3}\right) \delta_{i j}\right), \\
\rho_{11}\left(B_{3}\right)=\rho_{22}\left(B_{3}\right)=\frac{1}{e n} \cdot \frac{J\left(0,0, \mu^{\bullet}, T\right)}{J\left(0,1, B_{3}, \mu^{\bullet}, T\right) \Delta\left(B_{3}\right)^{\prime}}, \quad \rho_{33}(0)=\frac{1}{e n} \cdot \frac{J\left(0,0, \mu^{\bullet}, T\right)}{J\left(0,1, \mu^{\bullet}, T\right)^{\prime}} \\
R\left(B_{3}\right)=\frac{1}{z e n} \cdot \frac{J\left(0,0, \mu^{\bullet}, T\right) J\left(0,2, B_{3}, \mu^{\bullet}, T\right)}{J\left(0,1, B_{3}, \mu^{\bullet}, T\right)^{2} \Delta\left(B_{3}\right)}, \\
\left.=\frac{k}{z e} \cdot\left\{\frac{\left(\alpha_{i j}\left(B_{3}\right)\right)=\left(\alpha_{i i}\left(B_{3}\right) \delta_{i j}\right), \alpha_{11}\left(B_{3}\right)=\alpha_{22}\left(B_{3}\right)=}{J\left(0,1, B_{3}, \mu^{\bullet}, T\right)}-\mu^{\bullet}\right]+\frac{J\left(0,2, B_{3}, \mu^{\bullet}, T\right)^{2}}{J\left(0,1, B_{3}, \mu^{\bullet}, T\right)^{2}} \cdot\left[\frac{J\left(1,2, B_{3}, \mu^{\bullet}, T\right)}{J\left(0,2, B_{3}, \mu^{\bullet}, T\right)}-\mu^{\bullet}\right] \cdot B^{2}\right\} \frac{1}{\Delta\left(B_{3}\right)} \\
\left(\alpha_{33}(0)\right)=\frac{k}{z e} \cdot\left[\frac{J\left(1,1, \mu^{\bullet}, T\right)}{J\left(0,1, \mu^{\bullet}, T\right)}-\mu^{\bullet}\right], \\
N\left(B_{3}\right)=\left(\frac{k}{e}\right) \cdot \frac{J\left(0,2, B_{3}, \mu^{\bullet}, T\right)}{J\left(0,1, B_{3}, \mu^{\bullet}, T\right) \Delta\left(B_{3}\right)} \cdot\left[\frac{J\left(1,2, B_{3}, \mu^{\bullet}, T\right)}{J\left(0,2, B_{3}, \mu^{\bullet}, T\right)}-\frac{J\left(1,1, B_{3}, \mu^{\bullet}, T\right)}{J\left(0,1, B_{3}, \mu^{\bullet}, T\right)}\right],
\end{gathered}
$$

$$
\begin{gathered}
\left(\pi_{i j}\left(B_{3}\right)\right)=T \cdot\left(\alpha_{i j}\left(B_{3}\right)\right), \\
P\left(B_{3}\right)=T \cdot N\left(B_{3}\right), \\
\left(\chi_{i j}\left(B_{3}\right)\right)=\left(\frac{k}{z e}\right)^{2} \cdot T \cdot\left(X_{i j}\left(B_{3}\right)\right), \\
S(\vec{B})\left(\delta_{i j l} B_{l}\right)=\left(\frac{k}{z e}\right)^{2} \cdot T \cdot\left(\sum_{i j}\left(B_{3}\right)\right) .
\end{gathered}
$$

Formulas (14), (15) contain the cumbersome and difficult to analysis terms, thus they are presented here in their general forms and their exactly descriptions are not given in this work.

All formulas (8)-(15) have an analytical dependence on the general algorithmic functional - it is given as:

$$
\begin{gathered}
J\left(i, j, B_{3}, \mu^{\bullet}, T\right)=\int_{0}^{\infty}\left(\frac{\varepsilon}{k T}\right)^{i} \frac{u(\varepsilon)^{j}}{\delta\left(B_{3}\right)} G(\varepsilon)\left(-\frac{d f_{0}}{d \varepsilon}\right) d \varepsilon \\
\delta\left(B_{3}\right)=1+\left(u(\varepsilon) B_{3}\right)^{2},
\end{gathered}
$$

and on the dimensionless function:

$$
\Delta\left(B_{3}\right)=1+\frac{J\left(i, j, B_{3}, \mu^{\bullet}, T\right)}{J\left(i, j, B_{3}, \mu^{\bullet}, T\right)} B_{3}^{2} .
$$

This functional has the following evident properties:

$$
\begin{gathered}
J\left(i, j, B_{3}, \mu^{\bullet}, T\right)_{\left(u B_{3}\right) \gg 1}=\frac{1}{B_{3}^{2}} \int_{0}^{\infty}\left(\frac{\varepsilon}{k T}\right)^{i} u(\varepsilon)^{(j-2)} G(\varepsilon)\left(-\frac{d f_{0}}{d \varepsilon}\right) d \varepsilon=\frac{1}{B_{3}^{2}} J\left(i,(j-2), \mu^{\bullet}, T\right), \\
J\left(i, j, B_{3}, \mu^{\bullet}, T\right)_{\left(u B_{3}\right) \ll 1}=\int_{0}^{\infty}\left(\frac{\varepsilon}{k T}\right)^{i} u(\varepsilon)^{j} G(\varepsilon)\left(-\frac{d f_{0}}{d \varepsilon}\right) d \varepsilon=J\left(i, j, \mu^{\bullet}, T\right), \\
J\left(0,0, \mu^{\bullet}, T\right)=\int_{0}^{\infty} G(\varepsilon)\left(-\frac{d f_{0}}{d \varepsilon}\right) d \varepsilon=\int_{0}^{\infty} g(\varepsilon) f_{0} d \varepsilon=n\left(\mu^{\bullet}, T\right), \quad G(\varepsilon)=\int_{0}^{\infty} g(\varepsilon) d \varepsilon .
\end{gathered}
$$

Formulas (8)-(15), together with the functional (16) and function $\Delta\left(B_{3}\right)$, establish the general computational algorithms for the important kinetic properties of one energy valley crystals with an arbitrary isotropic dispersion law of charge carriers $\varepsilon_{\vec{p}}=\varepsilon(p)$ where these charge carriers are scattered by arbitrary defects of crystal lattice.

To the calculations of the properties (8)-(15), in a 
case of the classical strong magnetic fields, when the condition $\left(u B_{3}\right)^{2} \gg 1$ holds, the functional (17) should be used. On the other hand, in a case of the weak magnetic field, when the condition $\left(u B_{3}\right)^{2} \ll 1$ holds, the functional (18) should be used to these calculations. In this connection the formulas (8)-(15) may be called the determining formulas.

The determining formulas make possible the calculations of the kinetic properties of 2D crystals (nanosheets with a microscopic thickness) and 1D crystals (nanowires), where the space quantization of charge carriers' spectrum may occurs.

2.1 Kinetic properties of the isotropic crystals immersed in a strong magnetic field

A magnetic field with induction $B_{3}$ is called the classical strong if the condition $\left(u B_{3}\right)^{2} \gg 1$ holds, that is, $\delta\left(B_{3}\right)=1+\left(u B_{3}\right)^{2} \cong\left(u B_{3}\right)^{2}$. It is easy to show that the substition values of $\delta\left(B_{3}\right)=1+\left(u B_{3}\right)^{2} \cong\left(u B_{3}\right)^{2}$ into the appropriate functionals of different kinetic potentials and current carriers mobilities (8)-(15) gives the following formulas:

$$
\begin{gathered}
\rho_{11}=\rho_{22}=\frac{1}{e n} \cdot \frac{J\left(0,-1, \mu^{\bullet}, T\right)}{J\left(0,0, \mu^{\bullet}, T\right)}, \\
\rho_{33}(0)=\frac{1}{e n} \cdot \frac{J\left(0,0, \mu^{\bullet}, T\right)}{J\left(0,1, \mu^{\bullet}, T\right)^{\prime}} \\
R=\frac{1}{z e n^{\prime}} \\
\alpha_{11}=\alpha_{22}=\frac{k}{z e} \cdot\left[\frac{J\left(1,0, \mu^{\bullet}, T\right)}{J\left(0,0, \mu^{\bullet}, T\right)}-\mu^{\bullet}\right], \\
\alpha_{33}=\frac{k}{z e} \cdot\left[\frac{J\left(1,1, \mu^{\bullet}, T\right)}{J\left(0,0, \mu^{\bullet}, T\right)}-\mu^{\bullet}\right], \\
N\left(B_{3}\right) \sim\left(\frac{J\left(0,-1, \mu^{\bullet}, T\right)}{J\left(0,0, \mu^{\bullet}, T\right) B_{3}}\right)^{2}, \\
\pi_{i i}\left(B_{3}\right)=T \cdot \alpha_{i i}\left(B_{3}\right), \\
U_{H}\left(B_{3}\right)=U_{D}\left(B_{3}\right)=\frac{J\left(0,0, \mu^{\bullet}, T\right)}{J\left(0,-1, \mu^{\bullet}, T\right)} \\
\chi_{33}\left(B_{3}\right)=\chi_{33}(0) \\
\chi_{11}\left(B_{3}\right)=\chi_{22}\left(B_{3}\right) \sim\left(\frac{J\left(0,-1, \mu^{\bullet}, T\right)}{J\left(0,0, \mu^{\bullet}, T\right) B_{3}}\right)^{2}, \\
N\left(B_{3}\right), \\
{ }^{2},
\end{gathered}
$$

Hence it follows that in a strong magnetic field, the transverse magnetic resistance, Hall coefficient $R$, Seebeck coefficient, Hall mobility $U_{H}$, carrier mobility $U_{D}$ obtain their stationary values and the electron heat conductivity and coefficient of transverse NernstEttingshausen effect are inversely proportional to the square of magnetic induction.

Therefore, in strong magnetic fields, the known electron heat conductivity freezing effect occurs and the transversal Nernst-Ettingshausen effect is strongly reduced. In the same conditions, the Seebeck effect coefficient does not depend on the scattering mechanisms, but this coefficient depends on the energy spectrum structure. The Hall coefficient has the same independence from the scattering mechanisms.

2.2 Kinetic properties of the isotropic crystals immersed in a weak magnetic field, or when there is no any magnetic field

A magnetic field $B_{3}$ is called the weak magnetic field if it fulfils the condition $\left(u(\varepsilon) B_{3}\right) \ll 1$. Under this condition is easy to show that the determining formulas (8)-(15) for this case have the following form:

$$
\begin{gathered}
\rho\left(\mu^{\bullet}, T\right)=\frac{1}{e n} \cdot \frac{J\left(0,0, \mu^{\bullet}, T\right)}{J\left(0,1, \mu^{\bullet}, T\right)^{\prime}} \\
R\left(\mu^{\bullet}, T\right)=\frac{1}{z e n} \frac{J\left(0,0, \mu^{\bullet}, T\right) J\left(0,2, \mu^{\bullet}, T\right)}{J\left(0,1, \mu^{\bullet}, T\right)^{2}} \\
\alpha\left(\mu^{\bullet}, T\right)=\left(\frac{k}{z e}\right)\left[\frac{J\left(1,1, \mu^{\bullet}, T\right)}{J\left(0,1, \mu^{\bullet}, T\right)}-\mu^{\bullet}\right] \\
N\left(\mu^{\bullet}, T\right)=\left(\frac{k}{e}\right) U_{H}\left(\mu^{\bullet}, T\right)\left[\frac{J\left(1,1, \mu^{\bullet}, T\right)}{J\left(0,1, \mu^{\bullet}, T\right)}-\frac{J\left(1,2, \mu^{\bullet}, T\right)}{J\left(0,2, \mu^{\bullet}, T\right)}\right] \\
\pi\left(\mu^{\bullet}, T\right)=T \alpha\left(\mu^{\bullet}, T\right), \\
P\left(\mu^{\bullet}, T\right)=T N\left(\mu^{\bullet}, T\right), \\
\chi\left(\mu^{\bullet}, T\right)=\left(\frac{k}{e}\right)^{2} \frac{T}{\rho\left(\mu^{\bullet}, T\right)}\left[\frac{J\left(2,1, \mu^{\bullet}, T\right)}{J\left(0,1, \mu^{\bullet}, T\right)}-\left(\frac{J\left(1,1, \mu^{\bullet}, T\right)}{J\left(0,1, \mu^{\bullet}, T\right)}\right)^{2}\right] \\
U_{H}\left(\mu^{\bullet}, T\right)=\frac{J\left(0,2, \mu^{\bullet}, T\right)}{J\left(0,1, \mu^{\bullet}, T\right)}, \quad U_{D}\left(\mu^{\bullet}, T\right)=\frac{J\left(0,1, \mu^{\bullet}, T\right)}{J\left(0,0, \mu^{\bullet}, T\right)^{\prime}}
\end{gathered}
$$

These formulas show that a crystal being anisotropic by the action of a magnetic field, will turn into an isotropic crystal, when a magnetic induction vector $\vec{B}$ of this field will fulfil the condition $\left(u(\varepsilon) B_{3}\right) \ll 1$.

All algorithmic formulas (8a)-(15a) as well as (8b)(15b) have been calculated with the statistical methods by application of the non-equilibrium Gibbs grand canonical thermodynamic potential (3).

All these calculation were made under the following general conditions of observation: current carriers in a crystal have a reduced effective mass $m_{n}$ for electrons, or $m_{p}$ for holes, and these carriers in an isotropic crystal are characterized by the following energy dispersion law:

$$
\frac{p_{x}^{2}+p_{y}^{2}+p_{z}^{2}}{2 m m_{n}}=\frac{p^{2}}{2 m m_{n}}=E(\varepsilon)
$$

where $m$ is the electronic mass, the quantity $E(\varepsilon)$ is the quantum mechanical energy function of the first-order and it depends on the energy of current carriers in a crystal.

Under these general conditions of calculations, the general computational algorithmic functional for the kinetic properties of a crystal (18) has the following form: 


$$
J\left(i, j, \mu^{\bullet}, T\right)=U(r, T)^{j} N_{C}(T) I\left(i, j, \mu^{\bullet}, T\right),
$$

where the following notations are used:

$$
N_{C}(T)=\frac{8}{3 \sqrt{\pi}}\left(\frac{2 \pi m m_{n}}{h^{2}}\right)^{3 / 2},
$$

$U(r, T)$ - the temperature dependent function, it has a dimension of the mobility and it is described by the following formula:

$$
U(r, T)=\left[\frac{1}{T}\left(U_{A} \delta(0, r)+U_{O} \delta(1, r)+U_{I} \delta(2, r)\right)\right]\left(m_{n}\right)^{(r-5 / 2)} T^{(r-1 / 2)},
$$

where $U_{A}, U_{O}, U_{I}$ are the dimensional crystal constants, which depend on the nature of the crystal and the scattering mode of current carriers in the crystal lattice, $\delta(m, n)$ is the known Kronecker symbol, it has the following values: $\delta(m, n)=1$ for $m=n, \delta(m, n)=0$ for $m \neq n, r$ is called the scattering parameter and it has the following values: $r=0$ for the scattering by the acoustic phonons and point defects in the crystal lattice, $r=1$ for the scattering by the optical phonons when the crystal's temperature is larger than the Debye temperature, $r=2$ for the scattering by the charged impurities (ions) of the crystal lattice; and the dimensionless integral $I\left(i, j, \mu^{\bullet}, T\right)$ is given as:

$$
I\left(i, j, \mu^{\bullet}, T\right)=\int_{0}^{\infty} x^{i} \frac{E(x)^{(r j-j / 2+3 / 2)}}{\left(\frac{d E(x)}{d x}\right)^{2 j}}\left(-\frac{d f_{0}}{d x}\right) d x .
$$
$E_{G}$.

Next we apply this functional to calculation of the kinetic properties of isotropic crystals with narrow energy gaps band:

In these crystals, as a rule, the dispersion laws for electrons and holes are described by the Kane's nonparabolic

$$
\frac{p^{2}}{2 m_{m}}=E(\varepsilon)=\varepsilon+\frac{\varepsilon^{2}}{E_{G}}=\varepsilon+\beta(T) \frac{\varepsilon^{2}}{k T}, \quad \beta(T)=\frac{k T}{E_{G}} .
$$

For the dispersion relation (25) and actual scattering mechanisms, as were denoted by the scattering parameter $r$, the functional (24) has the form:

$$
I\left(i, j, \mu^{\bullet}, T\right)=\int_{0}^{\infty} x^{i} \frac{\left(x+\beta(T) x^{2}\right)^{(r j-j / 2+3 / 2)}}{\left(1+2 \beta(T) x^{2}\right)^{2 j}}\left(-\frac{d f_{0}}{d x}\right) d x .
$$

In this formula, $\beta(T)$ is the non-parabolicity parameter which in crystals with a wide energy gap $E_{G}$ can be far less than unity,thus the Kane's nonparabolic band (25) turns into the parabolic energy band. In this case, all calculations will be for crystals with the parabolic dispersion law.

Substituting the functional (21) with the dimensionless functional (26) into the algorithmic formulas (8a)-(15a) and (8b)-(15b), we obtain a set of calculation formulas for important kinetic properties of crystals with an isotropic nonparabolic energy band of current carriers.

In crystals with a wide energy gap $E_{G}$, the nonparabolicity parameter $\beta(T)<1$. In this connecton we expand the dimensionless functional $I\left(i, j, \mu^{\bullet}, T\right)(26)$ in a Taylor series of the little nonparabolicity parameter $\beta(T)=\frac{k T}{E_{G}}<1$ and we restrict ourselves to the linear term of this expansion. Thus, we have:

$$
\begin{gathered}
I\left(i, j, \mu^{\bullet}, T\right)=I\left(i, j, \mu^{\bullet}, T\right)_{(\beta=0)}+\left(\frac{d I\left(i, j, \mu^{\bullet}, T\right)}{d \beta}\right)_{\beta=0} \cdot \beta(T)+\ldots= \\
=F_{a(i, j)}\left(\mu^{\bullet}\right)+(a(i, j)-4 j-i) F_{(a(i, j)+1)}\left(\mu^{\bullet}\right) \cdot \beta(T)+\ldots=
\end{gathered}
$$

$$
=F_{a(i, j)}\left(\mu^{\bullet}\right)\left(1+(a(i, j)-i-4 j) \frac{F_{(a(i, j)+1)}\left(\mu^{\bullet}\right)}{F_{a(i, j)}\left(\mu^{\bullet}\right)} \beta(T)\right),
$$

where

$$
F_{a(i, j)}\left(\mu^{\bullet}\right)=\int_{0}^{\infty} x^{a(i, j)}\left(-\frac{d f_{0}}{d x}\right) d x
$$

Here, in these formulas, the function $F_{a(i, j)}\left(\mu^{\bullet}\right)$ with subscript

$$
a(i, j)=\left(\frac{3}{2}+i+r j-\frac{j}{2}\right)
$$

- this is known Fermi integral.

For non-degenerate current carriers the Fermi integral $(27 \mathrm{a})$ has the following forms: $F_{a(l, j)}\left(\mu^{*}\right)=$ 
$\Gamma(a(l, j)+1) e^{\mu^{*}}$, where $\Gamma(a(l, j)+1)$ is the gamma function, then in this case we have:

$$
I\left(i, j, \mu^{\bullet}, T\right)=\Gamma(a(i, j)+1)\left(1+(a(i, j)-i-4 j) \frac{\Gamma(a(i, j)+2)}{\Gamma(a(i, j)+1)} \beta(T)\right) \cdot e^{\mu^{\bullet}}
$$

In a case of a strong degeneracy $\left(\mu^{\bullet}>+4\right)$, the Fermi integral (27a) according to the Sommerfeld approximation, has the following form:

$$
F_{a(l, j)}\left(\mu^{\bullet}\right)=\int_{0}^{\infty} x^{a(l, j)}\left(-\frac{d f_{0}}{d x}\right) d x \approx\left(\mu^{\bullet}\right)^{a(l, j)}
$$

thus, the dimensionless functional (26) is given as:

$$
I\left(i, j, \mu^{\bullet}, T\right)=\left(\mu^{\bullet}\right)^{a(i, j)}\left(1+(a(i, j)-i-4 j) \mu^{\bullet} \beta(T)\right) .
$$

Formulas (28), (29) give the possibility of calculate the crystal kinetic properties in cases of the classical strong or weak magnetic field for the non-degenerate or strong degenerate current carriers with the nonparabolical Kane's dispersion law.

In a case of conducting crystals with a wide energy gaps $E_{G}$, the nonparabolicity parameter $\beta(T)=\frac{k T}{E_{G}} \ll 1$, that is, $\beta(T) \rightarrow 0$, Therefore, in such crystals the dispersion law will be a parabolic. Therefore, these formulas may be used for the crystals with a parabolic dispersion law, but the nonparabolicity parameter $\beta(T)$ should be set to zero, $\beta(T)=0$.

Budzhak Ya.S. - Professor, Doctor of Science;

Wactawski T. - Adjunct, Doctor of Philosophy in Physics.

[1] J.S. Budjak, Physics and Chemistry of Solid State 18(1), 7 (2017) (doi: 10.15330/pcss.18.1.7-14).

[2] Ya.S. Budzhak, T. Wacławski, Physics and Chemistry of Solid State 19(2), 134 (2018) (doi: 10.15330/pcss.19.2.134-138).

[3] Ya.S. Budzhak, T. Wacławski, Physics and Chemistry of Solid State 19(4), 303 (2018) (doi: 10.15330/pcss.19.4.303-306).

[4] Ya.S. Budzhak, L.O. Vasilechko, Foundations of statistical theory of thermal and kinetic properties of semiconductor crystals (Liga-Pres, Lviv, 2016).

[5] Ya.S. Budzhak, A.O. Druzhinin, T. Wacławski, Modern Statistical Methods of Investigations of Properties of Crystals as Micro- and Nanoelectronics Materials (Publishing House of Lviv Polytechnic, Lviv, 2018). 


\title{
Я.С. Буджак ${ }^{1}$, Т. Вацлавський ${ }^{2}$
}

\section{Важливі питання статистичної теорії теплових і кінетичних властивостей кристалів}

\author{
${ }^{1}$ Національний університет «Львівська політехніка», м. Львів, Україна, е-таil: jabudjak@ukr.net \\ ${ }^{2}$ Краківська політехніка, м. Краків, Польща, е-таil: tadeuszwaclawski00@gmail.com
}

В даній роботі приведені лаконічні розрахункові алгоритмічні формули теплових і кінетичних властивостей кристалів. Ці формули обгрунтовані для ізотропних кристалів 3 довільним законом дисперсії для носіїв струму, які розсіюються на довільних дефектах кристалічної гратки. В роботі ці алгоритмічні формули були використані для розрахунків важливих властивостей кристалів 3 непараболічним законом дисперсії Кейна для носіїв струму і описано шлях переходу до кристалів 3 параболічним законом дисперсії.

Ключові слова: потенціал Гіббса, хімічний потенціал, електропровідність, теплопровідність, тензор. 